ORIGINAL ARTICLE

\title{
Implementation of British Thoracic Society guidelines for acute exacerbation of chronic obstructive pulmonary disease: impact on quality of life
}

\author{
K Dheda, A Crawford, G Hagan, C M Roberts
}

Postgrad Med J 2004;80:169-171. doi: 10.1136/pgmj.2003.012831

Background: The British Thoracic Society (BTS) guidelines have not been examined collectively for their impact on chronic obstructive pulmonary disease (COPD). Whether intensive outpatient follow up of COPD patients after acute admission, using these guidelines, improved quality of life compared to the "usual practice" of primary care follow up was investigated.

Methods: Altogether 103 patients with a new diagnosis of COPD were admitted and screened over a four year period. Seventy patients were excluded because of another dominant medical condition or a mandatory requirement for intervention. Patients were randomised to regular primary care (control group, $n=15$ ) or chest clinic follow up (intervention group, $n=10$ ). Spirometry, oxygen saturation, St George's Respiratory Questionnaire (SGRQ), and Short Form 36 questionnaire were measured at baseline and six months. The intervention group was reviewed at least four times in the six month period and received spirometry, ambulatory oxygen assessment, smoking cessation advice, nebuliser assessment, a steroid trial, advice about nutrition/exercise, and introduction to a patient support group.

See end of article for authors' affiliations

Correspondence to: Dr K Dheda, c/o Dr C M Roberts, Chest Clinic, Whipps Cross University Hospital, Leytonstone, London E 11 1NR, UK; k.dheda@ucl.ac.uk

Submitted 17 July 2003 Accepted 14 August 2003
Results: There was no significant difference between baseline measurements in the two groups. There was a significant mean (SD) improvement in the SGRQ symptom score from baseline to six months in the intervention group [20.98 (20.36)] compared with the controls [0.23 (12.55)] $(p=0.004)$. At six months the SGRQ symptom score, impact score, and total score was significantly better in the intervention than the control group ( $p=0.01,0.02$, and 0.02).

Conclusion: Aggressive implementation of BTS guidelines after initial hospitalisation may improve respiratory health specific quality of life scores in patients with COPD. Larger studies are needed to confirm this finding.
C hronic obstructive pulmonary disease (COPD) is common and consumes vast healthcare resources. As the treatment of COPD is largely symptomatic, many management interventions are aimed at improving health related quality of life. The British Thoracic Society (BTS) management guidelines provide recommendations for optimum practice that include hospital follow up for those admitted with acute exacerbations and a number of potential therapeutic interventions. ${ }^{1}$ This advice is a consensus view and not based on grade A evidence. Some interventions-for example, pulmonary rehabilitation-are known to result in benefits to quality of life, ${ }^{2}$ but many other interventions have not been examined as a healthcare package for their impact on quality of life in COPD.

We undertook a study comparing hospital outpatient follow up, according to BTS guidelines, with the usual practice of primary care follow up in patients with stable COPD after discharge.

\section{METHODS}

Over a four year period (November 1996 to December 1999) 103 patients with a first admission of COPD were considered for the study. COPD was diagnosed if patients had progressive symptoms, a smoking history of $>20$ pack years, and a forced expiratory volume in one second $\left(\mathrm{FEV}_{1}\right)<80 \%$ of predicted with $\mathrm{FEV}_{1}$ /forced vital capacity ratio $<0.75$. Exclusions were another dominant medical condition, a mandatory reason for hospital follow up-for example, suspected cancer, already under outpatient follow up, or if they refused consent. Patients were randomised to regular outpatient follow up (intervention group) or primary care follow up (control group) for a six month period.

Demographic data, spirometry (including bronchodilator response), resting oxygen saturation, medical outcomes study Short Form 36, and St George's Respiratory Questionnaire (SGRQ) at baseline and six months, number of admissions, oxygen, and nebuliser prescriptions, and visits to an accident and emergency department over six months were recorded in all patients. For the SGRQ a change of four units or more was accepted as significant. ${ }^{34}$

A respiratory nurse and/or chest physician reviewed the intervention group at least four times in the six month period (at three, six, eight, 12 or 16 weeks). The following interventions were made at some or all of these visits: spirometry with reversibility, review of inhaler technique and peak flow diary, ambulatory oxygen assessment, smoking cessation advice, steroid trial, nebuliser assessments, review of medication for the addition of long acting B2 agonists and theophyllines, advice about nutrition and exercise, and introduction to a patient support group. Patients in the control group visited their primary care teams on a "need to" basis. Pulmonary rehabilitation was specifically excluded as it is already known to benefit such patients.

\section{Statistical methods}

The baseline and six month parametric data and scores were compared using Student's $t$ test.

Abbreviations: BTS, British Thoracic Society; COPD, chronic obstructive pulmonary disease; $\mathrm{FEV}_{1}$, forced expiratory volume in one second; SGRQ, St George's Respiratory Questionnaire 


\section{Ethical approval}

The Redbridge and Waltham Forest Research Committee gave ethical approval for this study.

\section{RESULTS}

\section{Baseline data}

Of the 103 patients screened, 70 did not satisfy the entry criteria, predominantly because of need for mandatory intervention or severe co-morbidity, and were excluded from the study. The remaining 33 patients were randomised to regular chest clinic follow up (intervention group) or primary care follow up (control group). Eight patients (five from the intervention and three from the control group) withdrew from the study resulting in 15 patients in the control group and 10 in the intervention group who completed the study. Patients withdrew from the control group because one patient was lost to follow up and two had repeated admissions and from the intervention group because two patients were lost to follow up, one inadvertently attended their general practitioner for follow up, and two patients had repeated hospital admissions. There were no significant differences between the two groups (control $v$ intervention) in the baseline quality of life scores, age $(71.33(8.39) v 68.40$ (5.76) years), $\mathrm{FEV}_{1}(39$ (11.88) $v 44.70$ (21.84) percent predicted), and severity of disease according to the BTS guidelines for COPD (20\% mild, 27\% moderate, and 53\% severe disease $v 20 \%$ mild, $20 \%$ moderate, and $60 \%$ severe disease).

\section{Symptom scores at six months}

The mean improvement of the SGRQ symptom score from baseline to six months in the intervention group (20.98 (20.36)) was significantly better compared with the control group (0.23 (12.55); $\mathrm{p}=0.004)$ (fig 1 ). In the intervention group, at six months the SGRQ symptom score, impact score (psychosocial function), and total score was significantly better than the control group ( $p=0.01,0.02$, and 0.02) (fig 2). Although there was a trend towards improvement in the Short Form 36 general health score $(p=0.067)$, this did not reach statistical significance.

There were fewer readmissions $(2 \vee 9)$ and patients with two or more exacerbations within a six month period $(2 \vee 3)$ in the intervention group but the small numbers precluded meaningful statistical analysis. There was no significant difference at six months in $\mathrm{FEV}_{1}$, resting oxygen saturations and the amount of oxygen, nebuliser, theophylline, and long acting bronchodilator prescriptions in the two groups.

\section{DISCUSSION}

This study suggests that regular follow up in the outpatient department accompanied by aggressive implementation of BTS management guidelines produces disease specific improvements in quality of life indicators but not in general health quality of life. Despite the enormous disease burden of COPD within the UK there are few studies addressing the

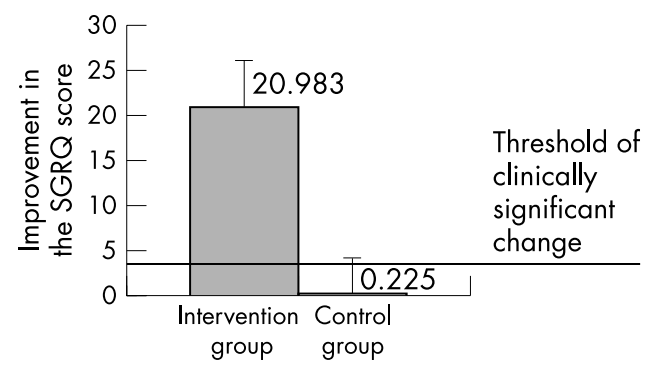

Figure 1 Mean change in the SGRQ score at six months in the control and intervention groups. Error bars represent SEM.

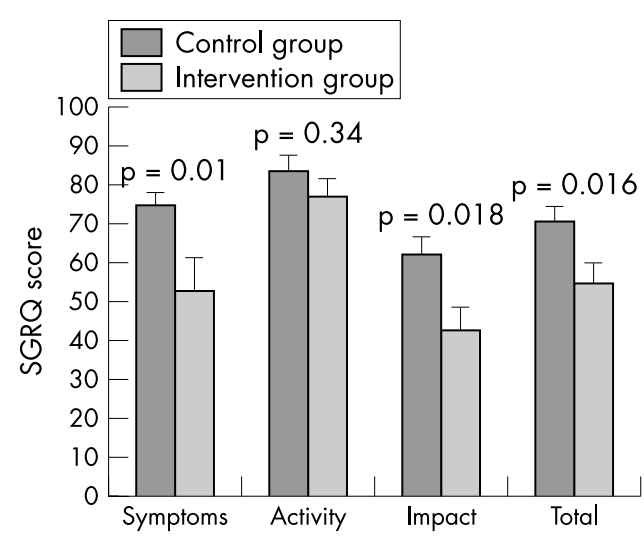

Figure 2 Mean six month SGRQ symptom, impact, activity, and total score in the control and intervention groups.

value of the BTS recommendations for active follow up of admitted patients. Indeed most of the intervention suggestions are individually unproven in this area and certainly have not been studied as a combined package of measures. The notable exception is the literature on pulmonary rehabilitation and this study purposefully excluded that intervention of proven value.

This current study is weakened by the small number of patients who completed the study. An important lesson can be learned from this. The small number enrolled was mainly due to the disease severity of the admitted patients that meant they could not ethically be randomised to the control group-for example, investigation of possible lung cancer and need for long term oxygen therapy. Others had severe comorbidity that dominated their illness profile and meant that COPD interventions may be ineffective. Finally, of those recruited a significant number withdrew because of concurrent illness and nine of 15 patients in the control group were readmitted within the six months of follow up period concordant with other research in this area.

The Short Form 36 scores did not improve to the same extent that the SGRQ scores did. This discrepancy may be explained because the Short Form 36 is a more generic instrument while the SGRQ is a more disease specific measure, with a much higher proportion of its content directly relevant to COPD. ${ }^{6}$

The observed change in the SGRQ could not be accounted for by changes in drug prescriptions or lung function. This was not surprising as the degree of correlation between objective measures of lung function and quality of life measures is inconsistent. ${ }^{7-10}$ As the symptoms of COPD are complex and multicausal it is possible that the secondary care package may have made an impact on aspects of COPD other than lung function. It may be that the simple effect of regular contact with a healthcare professional is beneficial and it remains unproven if any of the interventions made altered the pathophysiology of the COPD process or if simply the whole package of care provided a placebo impact.

This report is noteworthy for two reasons. Firstly it objectively justifies the consensus derived recommendations for a package of measures to be allocated to patients discharged after a first admission with COPD. Such an allocation of secondary care resources would, however, be subject to a consideration of the cost effectiveness of this approach, an aspect not examined in this study. Secondly it highlights the surprising paucity of literature on this important subject. Larger multicentre studies are needed to confirm our findings and to identify the individual interventions that may account for a change in quality of life indices and the mechanisms by which this is achieved. 


\section{Authors' affiliations}

K Dheda, A Crawford, G Hagan, C M Roberts, Whipps Cross University Hospital, London, UK

Conflicts of interest: $\operatorname{Dr} G$ Hagan worked as an Honorary Clinical Assistant at Whipps Cross Hospital while in the employ of Glaxo Welcome at the time of the study.

\section{REFERENCES}

1 Anonymous. BTS guidelines for the management of chronic obstructive pulmonary disease. The COPD Guidelines Group of the Standards of Care Committee of the BTS. Thorax 1997;52:S1-28.

2 Bendstrup KE, Ingemann Jensen J, Holm S, et al. Out-patient rehabilitation improves activities of daily living, quality of life and exercise tolerance in chronic obstructive pulmonary disease. Eur Respir J 1997:10:2801-6.

3 Jones PW. Quirk FH. Baveystock CM. The St George's Respiratory Questionnaire. Respir Med 1991:85:S25-31.

4 Quirk FH, Baveystock CM, Wilson R, et al. Influence of demographic and disease related factors on the degree of distress associated with symptoms and restrictions on daily living due to asthma in six countries. Eur Respir J 1991;4:167-71.

5 Roberts CM, Lowe D, Bucknall CE, et al. Clinical audit indicators of outcome following admission to hospital with acute exacerbation of chronic obstructive pulmonary disease. Thorax 2002;57:137-41

6 Yusen R. What outcomes should be measured in patients with COPD? Chest $2001 ; 119: 327-8$

7 Redelmeier DA, Goldstein RS, Min ST, et al. Spirometry and dyspnea in patients with COPD. When small differences mean little. Chest 1996; 109:1163-8.

8 Mahler DA, Tomlinson D, Olmstead EM, et al. Changes in dyspnea, health status, and lung function in chronic airway disease. Am J Resp Crit Care Med 1995; 151:61-5.

9 Ferrer M, Alonso J, Morera J, et al. Chronic obstructive pulmonary disease stage and health-related quality of life. The Quality of Life of Chronic Obstructive Pulmonary Disease Study Group. Ann Intern Med 1997; 127:1072-9.

10 Ries AL, Kaplan RM, Limberg TM, et al. Effects of pulmonary rehabilitation on physiologic and psychosocial outcomes in patients with chronic obstructive pulmonary disease. Ann Intern Med 1995;122:823-32.

\section{IF ONLY I'D KNOWN}

\section{Ten things I wish I'd known when I was 25}

(1) That everyone who tries to live forever eventually dies in the attempt.

(2) That older folk-particularly those in charge-don't do what they do simply because they have lost their marbles along the way.

(3) That giving patients what they want rather than what they really need will save you a lot of heartache.

(4) Save your energy only for the few battles you could actually win.

(5) Find out as soon after the start of the consultation begins as possible what the patient wants from you-it's rarely what you imagine they should want.

(6) Very rarely is the patient's view of themselves or their problem anywhere near shared by what those who know them well. Canvass a variety of views before accepting the patient's version.

(7) That excellence in academic performance is rarely rewarded with popularity-but is worth it anyway.

(8) That in a profession where salaries are all the same-relatively small differences in advantage seem to matter hugely and cause massive envy. Try to hide your success as much as possible, or if uncovered, deny it.

(9) That there is much more to death than medical failure and that there is much more to life than being a doctor.

(10) That doctors not only alarmingly rarely really know what's best for the patientthey don't know what's best for doctors-take all career advice with a pinch of lithium. 